\title{
Does perceptual adaptation to telestereoscopically enhanced depth depend on the recalibration of binocular disparity?
}

\author{
S. KAY FISHER and SHELDON M. EBENHOLTZ \\ University of Wisconsin, Madison, Wisconsin
}

\begin{abstract}
Apparent depth is significantly reduced after viewing a rotating three-dimensional form through a system of mirrors which enhances binocular disparity by optically increasing the separation between the eyes. The change in depth perception has previously been interpreted in terms of the adaptive recalibration of binocular disparity by kinetic sources of depth information. The present experiments showed that the same result is still obtained when either disparity or kinetic depth is absent during the exposure interval. The depth aftereffect failed to occur, however, when artificial pupils were used during binocular observation of the rotating form through the mirrors. These results demonstrate that the conflict between disparity and kinetic depth is neither necessary nor sufficient to produce the adaptive change in depth perception. A close association between depth and distance aftereffects was consistently found, suggesting that both types of perceptual change derive from the same underlying process. The accommodation system was implicated by the fact that the perceptual aftereffects were present following monocular exposure, but were absent under the artificial pupil exposure condition.
\end{abstract}

Wallach, Moore, and Davidson (1963) were the first to study the modification of stereoscopic depth perception following optical enhancement of the separation between the eyes. An arrangement of mirrors called a telestereoscope was used to effect a more than twofold increase in interpupillary distance. Since binocular disparity depends on the distance between the eyes, the device increased horizontal disparities by a corresponding factor. Wallach et al. had subjects judge the apparent depth of a stationary three-dimensional form (e.g., a cube) viewed through the telestereoscope. The perceived depth of the object was about twice its actual depth, in accordance with the enhanced binocular disparity. The form was then set into rotation about a vertical axis and subjects observed it through the device for a period of $10 \mathrm{~min}$. When the tests on the stationary form were repeated, a substantial reduction in its apparent depth was found to have occurred. The change was adaptive in that it acted to partially restore veridical depth perception for objects seen through the telestereoscope. The adaptation amounted to about $20 \%$ of that theoretically possible, based on complete adaptation for the enhanced disparity.

Wallach et al. (1963) interpreted their results in terms of a form of perceptual learning elicited by conflicting cues to depth. With a moving object, such as the rotating form, the continuous perspective changes in the twodimensional retinal projection provide a powerful source

This research was supported in part by Grant No. EY03421 from the National Institutes of Health to the second author. We thank K. Ciuffreda and $W$. Shebilske for their critical reading of an earlier version of the manuscript, and Steven Carlson for drawing the figures.

The authors' current address is: SUNY/State College of Optometry, 100 East 24th Street, New York, NY 10010. of depth information. The experience of three-dimensional depth arising from this type of transformation in the stimulus is known as the kinetic depth effect, or KDE (Wallach \& O'Connell, 1953). Since the KDE is a monocular depth cue, it is unaltered by the telestereoscope and therefore is placed in direct conflict with the depth information provided by binocular disparity. According to cueconflict theory, the pairing between the two discrepant cues results in the recalibration of disparity by the KDE such that a unit amount of disparity gives rise to less apparent depth than was perceived prior to exposure to the telestereoscope. ${ }^{1}$

Alternatively, it is possible that changes in distance perception, rather than recalibration of the disparity-depth relationship, are responsible for adaptation to the telestereoscope. Since the perceptual system must take distance into account in deriving depth from disparity, changes in the registration of distance would result in secondary aftereffects of apparent depth. Wallach et al. (1963) argued against such an interpretation of the results of their experiments on the grounds that the depth aftereffect found following exposure to the telestereoscope was not accompanied by a reduction in apparent size.

Unfortunately, the assumption that changes in the perception of distance must necessarily give rise to aftereffects of both size and depth may have been incorrect. Since apparent size is proportional to distance, whereas apparent depth is approximately proportional to distance squared, an alteration in registered distance would be expected to produce a much larger change in the perception of depth than of size. This has been verified empirically in the context of adaptation to optical distortion of distance (Wallach, Frey, \& Bode, 1972). If a relatively 
small change in registered distance were induced during exposure, the size aftereffect might be undetectable. Clearly, it would be better to carry out tests of distance perception, rather than to rely completely on the indirect size-judgment task.

Oculomotor sources of distance information, that is, vergence and accommodation, play an important role in the processing of depth from disparity (Ono \& Comerford, 1977; Ritter, 1979; Wallach \& Zuckerman, 1963). Changes within one or more of these systems may therefore be expected to lead to the modification of stereoscopic depth perception. Given plasticity in the neural control of vergence and accommodation (Ebenholtz, 1983, 1985; Ebenholtz \& Fisher, 1982), perceptual learning (Wallach, 1976) need not occur to account for adaptation to the telestereoscope.

In the telestereoscope situation, a reduction in registered distance would be required to produce the observed aftereffect in perceived depth. Conditions of oculomotor conflict existed during the exposure interval in Wallach et al.'s (1963) study, which may have facilitated some form of oculomotor adaptation. The authors stated that the degree of convergence required to view the rotating form through the telestereoscope was the same as that which would be required to view it directly. However, given the optical enhancement of the observer's interpupillary distance, the angle of one set of mirrors can be adjusted so that convergence angle is the same as without the device for no more than one point in space, with locations nearer or farther away requiring greater or less than normal convergence, respectively (Figure 1).

Since a three-dimensional object was viewed through the mirrors and fixation was uncontrolled, convergence angle was undoubtedly altered, relative to normal viewing conditions, throughout much of the exposure inter$\mathrm{val}$. In contrast, except for a slight increase in optical distance (about 0.15 D) due to the greater length of the pathway of light to the eyes, the stimulus to accommodation remained the same as without the telestereoscope. Accommodation and convergence therefore had to be dissociated to some degree in order to maintain both fusion and sharp focus. ${ }^{2}$ No methods currently exist for estimating how innervational balance between the accommodation and vergence controllers (see systems model, Ebenholtz \& Fisher, 1982) is achieved under conditions in which the stimuli for accommodation and convergence are noncongruent and vary over time. It is entirely possible that the oculomotor demand created within these systems was conducive to a change in active innervation, such that the test form was experienced as both nearer and reduced in depth following exposure.

The present experiments demonstrated that the conflict between disparity and the KDE during exposure to the telestereoscope is neither necessary (Experiments 1 and 2) nor sufficient (Experiment 3) to produce a significant reduction in binocular apparent depth. Moreover, the experiments showed that the depth aftereffect can be ac-

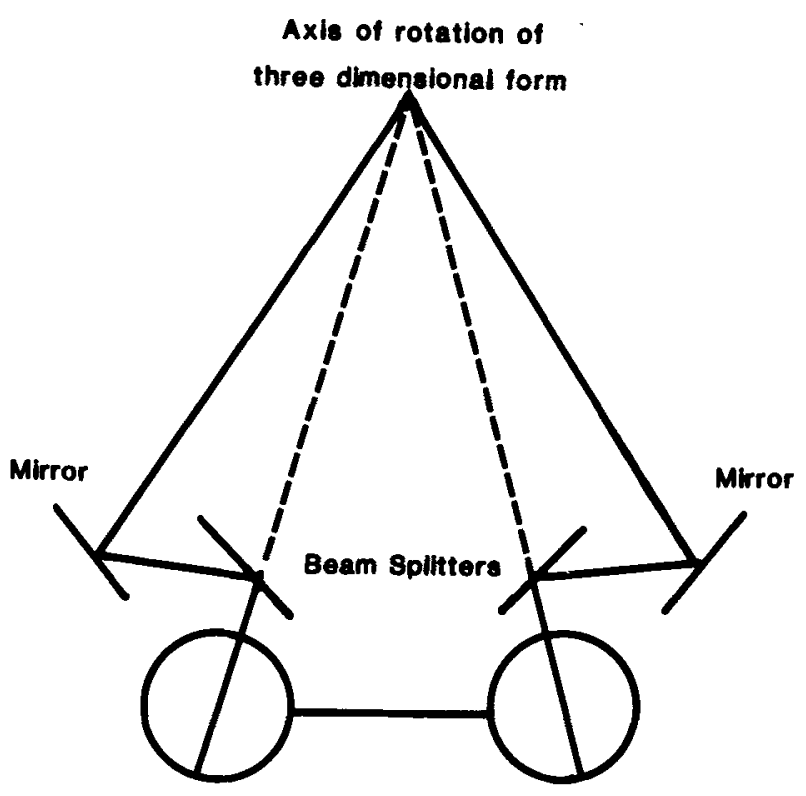

Figure 1. The telestereoscope, showing the pathway of the reflected light (solid lines) to the observer's eyes. Dashed lines represent the pathway that would be taken with direct viewing. Note that the beam splitters have been rotated slightly and are not exactly parallel to the outer mirrors. Making the reflected and direct images congruent makes the convergence angle required for fixation on the axis of rotation of the three-dimensional form the same as that needed without the telestereoscope.

counted for in terms of changes in distance perception that apparently derive from some type of oculomotor change related to the accommodation system. In Experiment 1, the effects of observing a rotating three-dimensional form through the telestereoscope under binocular and monocular viewing conditions were compared. Changes in stereoscopic depth perception following monocular exposure to the device cannot reflect recalibration of binocular disparity by the $\mathrm{KDE}$, because no disparity, and hence no cue conflict, is present during the inducing period.

\section{EXPERIMENT 1}

\section{Method}

Design. The two telestereoscopic viewing conditions, standard and monocular, were tested in a within-subjects design. In the standard exposure condition, the subject viewed the rotating object through the mirrors of the telestereoscope with both eyes. In the monocular condition, the nondominant eye (as judged with a pointing test) was occluded during the exposure interval. Separate sessions separated by a minimum of $24 \mathrm{~h}$ were used for the two exposure conditions. The order of conditions was chosen randomly for each subject. To reduce the variability between subjects, a selection criterion was employed: subjects had to show a pretest to posttest reduction in apparent depth of $0.70 \mathrm{~cm}$ or better under the standard condition to be included in the final sample.

Apparatus. The telestereoscope consisted of two mirror and beam splitter combinations, one for each eye, mounted on an optical bench parallel to the subject's frontal plane (Figure 1). A cover attached to the side of each beam splitter farthest from the subject ensured that objects could be viewed only along the reflected pathway from 
the mirrors. The centers of the mirror and beam splitter on each side were separated by approximately $3.8 \mathrm{~cm}$; the angle of the beam splitters was adjusted somewhat for individual subjects to ensure that the convergence angle required for the axis of rotation of the three-dimensional form would be the same as that required for viewing directly without the telestereoscope.

The exposure and test forms were presented straight ahead, centered in the subject's median plane. An adjustable base with a small motor inside was placed on a second bench for this purpose. A metal rod $24 \mathrm{~cm}$ in length and $1.3 \mathrm{~cm}$ in diameter, with an adjustable marker $(4.4 \times 2.5 \mathrm{~cm})$ to be used for the kinesthetic judgments of apparent depth, was mounted on the front side of the shelf facing the subject (Figure 2). The rod was positioned parallel to the optical bench holding the telestereoscope and could be reached easily but not seen by the subject when his/her head was placed in the chin and forehead rest. The clamp securing the left-hand end of the rod to the shelf served as a stop for the marker.

Two different three-dimensional forms were used, a cube for the exposure interval and a pyramid for the depth judgments. The forms were constructed from balsa wood, each stick being $0.2 \mathrm{~cm}$ wide on each of its four faces and painted flat black. The cube measured $4.3 \mathrm{~cm}$ on each side and was oriented so that a diagonal running through its center would form an extension of the vertical supporting stalk (Figure 3 ). The pyramid measured $4.7 \mathrm{~cm}$ from apex to base. The vertical supporting stalk was attached at the center of one of the edges of the 4.7-cm-square base so that the two were coplanar (Figure 3). A curved sheet of white cardboard was placed $75 \mathrm{~cm}$ from the subject to serve as a homogeneous background.

Distance tests were carried out with two types of targets: the threedimensional pyramid (Figure 3 ) described above and a luminous two-dimensional target which was identical to that used by Ebenholtz and Fisher (1982) and which was seen in total darkness. A Green's refractor was adjacent to the table so that the refractor head could easily be positioned in line with a target mounted on the optical bench.

Procedure. The subject's interocular axis, measured with a Titmus PD scope, and eye dominance were recorded prior to the pretests. Lateral phoria was then measured with the Green's refractor with a Maddox rod, using an intermittent exposure technique (Ebenholtz \& Fisher, 1982). The target consisted of a red light-emitting diode (LED) with cross hairs, mounted at eye level at a distance of $33.3 \mathrm{~cm}$ from the front surface of the cornea and seen in total darkness.

Following the phoria testing, the refractor head was replaced by a chin and forehead rest and the subject was instructed in an openloop distance judgment task. The index finger of the preferred hand was placed in a thimble-like well which the subject would slide along

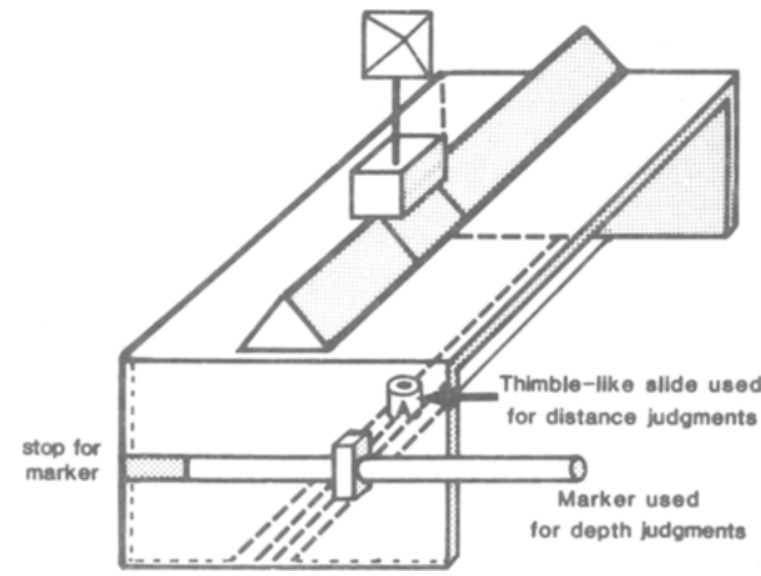

Figure 2. Apparatus used for kinesthetic judgments of apparent depth and distance. The three-dimensional pyramid used as one of the test targets is shown in position on the optical bench.
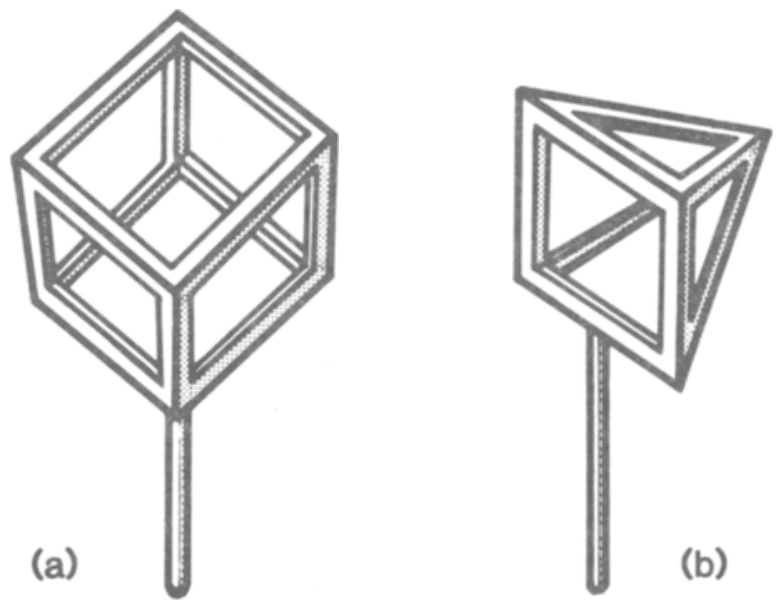

Figure 3. Three-dimensional forms used for the exposure interval (a) and depth judgments (b). The cube was seen while rotating. The pyramid was always oriented so that its base was parallel to the observer's frontal plane.

a track on the table below the optical bench where the target was mounted (Figure 2). A pointed metal indicator could be felt with the tip of the finger inside the bottom of the well. The subject's task was simply to slide the well along the track so that the felt position of the pointed indicator inside it matched the visual position of the two-dimensional luminous target. The center of the target was in the median plane and was located at eye level. Two practice trials were given, using binocular viewing and target distances of 29 and $37 \mathrm{~cm}$, in that order. Two binocular test trials and two monocular test trials followed, with the target at $33.3 \mathrm{~cm}$ from the eyes.

Throughout the entire series of distance tests, beginning with the practice trials, the starting position of the slide was alternately set $10 \mathrm{~cm}$ nearer and $10 \mathrm{~cm}$ farther away than the target. Half the subjects began the series with the nearer starting position and half with the farther starting position. The mean of the two settings for the test trials at $33.3 \mathrm{~cm}$ served as the subject's distance score in each viewing condition (monocular or binocular).

When the distance tests for the two-dimensional target were completed, the subject's chair was rotated $90^{\circ}$ in order to face the viewing position for the telestereoscope. The base of the pyramid target was placed $33.3 \mathrm{~cm}$ from the eyes and oriented parallel to the subject's frontal plane. The apex of the pyramid, pointing away from the subject, was at eye level. While binocularly viewing the pyramid, the subject made two judgments each of the distance of the form, its depth from base to apex, and the size of the base from left to right, in that order. For the distance tests, the subject fixated the base of the pyramid and matched its distance with the unseen hand by moving the slide or well located in the track on the table below. The distance task was essentially the same as that used for the two-dimensional target, and the subjects required little instruction.

For the depth and size tests, the subject moved the marker along the metal rod (Figure 2) with the unseen hand so that the length of the rod felt between the stop and the nearest edge of the marker corresponded to the distance in space separating the base of the pyramid from its apex (depth test) or its width from left to right (size test). The index finger of the preferred hand was slid continuously back and forth along the rod in making the match. One of the two trials began with the marker located $0 \mathrm{~cm}$ from the stop, and the other with the marker located $15 \mathrm{~cm}$ from the stop. The mean of the two settings served as the subject's depth or size score. Starting position was counterbalanced across subjects.

When the pretests were completed, the pyramid was replaced by the cube (to be used as the exposure target). The cube was located so that its central axis of rotation was $33.3 \mathrm{~cm}$ from the subject's 
eyes. If the monocular exposure condition was to be run, an eye patch was placed over the nondominant eye. The subject then observed the rotating cube for a period of $10 \mathrm{~min}$. The cube rotated in the clockwise direction (as seen from above) at a rate of $4 \mathrm{rpm}$. During the exposure interval, the subject was encouraged to describe the appearance of the form.

Following the exposure period, the subject sat with eyes closed while the rotating cube was replaced by the stationary pyramid. The procedure used for the pretests was then replicated for the posttests, with the exception of some changes in the order of presentation. The depth tests were completed first, followed by the distance and size tests. The latter tests were carried out in the following order: binocular distance and then size judgments with the base of the pyramid as the target, binocular distance judgments with the twodimensional target, and monocular distance judgments with the same target. A posttest phoria measurement was taken after all the perceptual tests.

Subjects. Five female and 3 male undergraduate students, ranging from 18 to 21 years of age, served as subjects in exchange for extra credit in the psychology course in which they were enrolled. All the subjects had acuity of $20 / 25$ or better in each eye as measured with a Bausch and Lomb Master Orthorater. None had ever worn glasses or contact lenses for any reason. Because of the relatively high criterion set for the magnitude of the depth aftereffect under the standard condition, the subjects selected may all be considered high adaptors. Fourteen additional subjects were dismissed from the experiment due to a variety of problems. Six subjects failed to experience any depth enhancement for the pyramid target on the pretests; they saw little or no difference between its size from left to right and its extent from front to back. For at least three of these subjects, this was due to a lack of fusion of the images for the two eyes, since diplopia was clearly present. Four other subjects were dismissed as a result of atypical phoria scores on the pretest. Of these, three showed varying degrees of esophoria at near $(+1$ to +6 PD), whereas exophoria is the norm (Duke-Elder \& Wybar, 1973), and one showed extreme exophoria ( -20 PD). The remaining four subjects failed to meet the criterion for depth adaptation un- der the standard exposure condition: one showed a negligible reduction in apparent depth and the other three showed a reversed aftereffect.

\section{Results}

The data for each of the perceptual tests and for lateral phoria are summarized in Table 1 . The pretests using the binocularly viewed three-dimensional pyramid indicated that the two conditions were equivalent with respect to the judged depth $[t(7)=-0.12, p>.05]$, distance $[t(7)$ $=-1.03, p>.05]$, and size $[t(7)=-1.35, p>.05]$ of the target prior to exposure. Similarly, there were no significant pretest differences between the two conditions for either the binocular $[t(7)=-0.48, p>.05]$ or monocular $[t(7)=1.11, p>.05]$ distance judgments using the two-dimensional target, or for lateral phoria $[t(7)$ $=-0.97, p>.05]$.

Analysis of the depth aftereffect scores indicated that a significant pretest to posttest reduction in apparent depth occurred under each condition. It was especially noteworthy that every subject, without exception, perceived the pyramid as shallower following monocular observation of the rotating form through the telestereoscope. The mean change for the monocular observation condition $(-1.04 \mathrm{~cm})$ was comparable in magnitude to that produced under the standard condition $(-1.24 \mathrm{~cm})$; the difference was statistically nonsignificant $[t(7)=-1.12$, $p>.05]$. Clearly, cue conflict is not necessary to produce the adaptation phenomenon.

For the distance aftereffects, the pattern of results across conditions was somewhat different for the various types

Table 1

Means and Standard Errors for Measurements in Experiment 1

\begin{tabular}{|c|c|c|c|c|c|c|}
\hline \multirow[b]{2}{*}{ Test Type } & \multicolumn{2}{|c|}{ Pretest } & \multicolumn{2}{|c|}{ Posttest } & \multicolumn{2}{|c|}{$\Delta$} \\
\hline & Mean & $S E$ & Mean & $S E$ & Mean & $S E$ \\
\hline \multicolumn{7}{|c|}{ Standard Exposure } \\
\hline Depth & 10.21 & 1.03 & 8.97 & 1.00 & $-1.24^{*}$ & 0.15 \\
\hline $\begin{array}{l}\text { Binocular Distance } \\
\text { (Pyramid) }\end{array}$ & 38.63 & 1.31 & 36.53 & 0.97 & $-2.10^{*}$ & 0.98 \\
\hline $\begin{array}{l}\text { Binocular Distance } \\
\text { (Two-dimensional Target) }\end{array}$ & 37.72 & 0.91 & 36.49 & 0.84 & -1.23 & 1.53 \\
\hline $\begin{array}{l}\text { Monocular Distance } \\
\text { (Two-dimensional Target) }\end{array}$ & 40.75 & 1.70 & 36.29 & 1.71 & $-4.46^{*}$ & 0.37 \\
\hline Size & 5.32 & 0.40 & 5.80 & 0.64 & 0.48 & 0.30 \\
\hline Phoria & -9.00 & 1.59 & -7.63 & 2.30 & 1.38 & 1.05 \\
\hline \multicolumn{7}{|c|}{ Monocular Exposure } \\
\hline Depth & 10.27 & 0.88 & 9.23 & 0.86 & $-1.04 *$ & 0.11 \\
\hline $\begin{array}{l}\text { Binocular Distance } \\
\text { (Pyramid) }\end{array}$ & 39.63 & 1.52 & 37.70 & 1.28 & $-1.93 *$ & 0.49 \\
\hline $\begin{array}{l}\text { Binocular Distance } \\
\text { (Two-dimensional Target) }\end{array}$ & 38.30 & 1.35 & 38.21 & 1.86 & -0.09 & 0.89 \\
\hline $\begin{array}{l}\text { Monocular Distance } \\
\text { (Two-dimensional Target) }\end{array}$ & 38.91 & 1.73 & 38.77 & 2.13 & -0.14 & 1.21 \\
\hline Size & 5.87 & 0.42 & 6.04 & 0.57 & 0.17 & 0.31 \\
\hline Phoria & -7.63 & 2.12 & -9.63 & 2.22 & -2.00 & 1.34 \\
\hline
\end{tabular}

*Significant at the $p=.05$ level or better. Apparent depth, distance, and size scores are in centimeters. Phoria scores are in prism diopters; negative phoria scores indicate exophoria. Aftereffect values $(\Delta)$ are derived from the (posttest - pretest) scores for each subject. 
of tests. The conditions were equivalent when the pyramid was used as the target $[t(7)=-0.19, p>.05]$; a significant reduction in apparent distance occurred in each case. The fact that the depth aftereffect was accompanied by a significant reduction in the apparent distance of the three-dimensional test target also suggests that factors other than the recalibration of binocular disparity are responsible for adaptation to the telestereoscope. When an object of constant disparity is experienced as nearer than before, the same perceptual processing that contributes to depth constancy under normal circumstances must lead to a reduction of apparent depth. With the twodimensional target and binocular testing, no differences in the aftereffect scores were found across conditions $[t(7)$ $=-1.83, p>.05]$. Although the distance aftereffects were in the same direction as those found with the threedimensional target, none differed significantly from zero. When testing was carried out monocularly with the twodimensional target, the distance aftereffect in the standard exposure condition was significantly larger than that in the monocular exposure condition $[t(7)=-2.46, p \leq$ $.05]$. The reduction in monocular apparent distance differed significantly from zero in the standard condition $[t(7)=-12.05, p \leq .05]$, in which the magnitude was large, averaging $-4.46 \mathrm{~cm}$.

The exposure conditions did not differ with respect to changes in apparent size $[t(7)=0.97, p>.05]$ or phoria $[t(7)=1.78, p>.05]$. These aftereffects were nonsignificant in each case. The absence of a significant reduction in apparent size cannot be taken as critical evidence against the idea that the depth aftereffect results from an underlying change in the registration of distance. As noted previously, because apparent size is directly proportional to distance, whereas depth is approximately proportional to distance squared, a larger change in distance perception may be needed to produce an aftereffect of size than to produce one of depth. The depth aftereffect expected on the basis of the actual pretest to posttest reduction in the perceived distance of the pyramid would amount to $-1.09 \mathrm{~cm}$ and $-0.62 \mathrm{~cm}$ for the standard and monocular exposure conditions, respectively. In contrast, the expected size aftereffect would be only $-0.27 \mathrm{~cm}$ for the standard exposure condition and $-0.19 \mathrm{~cm}$ for the monocular exposure condition. As can be seen in Table 1, changes in apparent size of that order of magnitude would be less than the corresponding error of measurement in each case. The absence of a significant pretest to posttest shift in measured phoria in either condition indicates that changes in fusional tonus were not produced.

\section{EXPERIMENT 2}

In Experiment 2, the depth aftereffect produced in the absence of cue conflict between disparity and kinetic depth was again compared with that produced using standard exposure to the telestereoscope. However, this time kinetic depth information, rather than binocular disparity, was the cue eliminated under the no-conflict condition.
This was accomplished by using binocular observation of the stationary form through the mirrors (static condition). The manipulation of exposure conditions was therefore complementary to that used previously. Finding a significant depth aftereffect under the no-conflict condition would again argue against the recalibration of disparity by kinetic depth as the process responsible for the perceptual change.

In the third condition, subjects viewed the rotating form binocularly through the mirrors of the telestereoscope, but with the addition of small artificial pupils before each eye. By increasing the depth of focus, artificial pupils effectively reduce the need for active, blur-driven accommodation. If the perceptual aftereffects depend on the accommodative demand normally produced by the mirrors, no change in apparent depth or distance should be found under the artificial-pupil condition. In contrast, recalibration theory would predict equivalent aftereffects under the standard condition and the artificial-pupil condition, since both would involve the same conflict between binocular disparity and kinetic depth.

\section{Method}

Design. Three exposure conditions-kinetic, static, and kinetic with artificial pupil-were tested in a within-subjects design. The kinetic condition corresponded to the standard condition used in Experiment 1. The static condition differed in that subjects viewed the stationary form through the mirrors of the telestereoscope during the exposure interval. For the artificial-pupil condition, the form rotated during exposure, but was observed through small apertures in front of each eye. Binocular viewing was used for each of the three exposure conditions. A separate session was required for each of the three conditions; successive sessions were separated by a minimum of $24 \mathrm{~h}$. The order of the conditions was randomized separately for each subject.

Procedure. The procedure was identical in all respects to that used in the previous experiment. For the artificial pupils, two discs were cut from an aluminum sheet and a hole $2 \mathrm{~mm}$ in diameter was bored in the center of each. ${ }^{3}$ The discs were appropriately sized to be fit into a standard pair of optometric trial frames in place of lenses. For consistency, the trial frames were also worn during the exposure interval in the other two conditions, but with 0.0 spherical diopter (SD) lenses inserted in place of the discs.

For the artificial-pupil condition, care had to be taken to align the apertures properly so that the entire form could be seen with each eye and fusion could be easily obtained. The trial frames were first adjusted so that the distance between the eyes and the discs was reduced as much as possible. The initial separation between the centers of the discs was determined by the size of the subject's interocular axis, as previously measured with the Titmus PD scope. Fine adjustment was then carried out in the following manner. A rod of the same size and diameter as the supporting stalk of the exposure form was placed $33.3 \mathrm{~cm}$ from the eyes. One eye was then occluded, and the aperture in front of the fixating eye was moved slightly to the left or right as needed for an unobstructed view of the rod. In most cases, only very small changes were required. The procedure was then repeated with the other eye as the viewing eye. After the rod was replaced by the rotating form, the subject was asked to close and open each eye in succession as a final check to make sure that the entire form was visible in each monocular view. ${ }^{4}$

The criterion for depth adaptation in the kinetic condition was the same as that in the preceding experiment. Subjects had to show 
a pretest to posttest reduction in apparent depth of at least $0.70 \mathrm{~cm}$ in the kinetic condition in order to be included in the final sample.

Subjects. Five female and 3 male undergraduate students ranging from 18 to 23 years of age served as subjects. Twelve additional subjects were dismissed or replaced for a variety of reasons. Six of these subjects showed either esophoria or orthophoria during the pretests on the first session, whereas exophoria is the norm at near distances. One subject had very poor acuity $(20 / 100)$ in one eye. Two others had difficulty with the pretest judgments. One of these subjects simply could not achieve fusion of the images for the two eyes, and one failed to experience the form as threedimensional (i.e., as having any extension in depth). The remaining 3 subjects were replaced because they showed a pretest to posttest increase in apparent depth on the session in which kinetic exposure was used.

\section{Results}

The data for each of the three exposure conditions are summarized in Table 2 . No significant differences were found between conditions with respect to the initial judgments of the depth $[F(2,14)=0.71, p>.05]$, distance $[F(2,14)=1.22, p>.05]$, or size $[F(2,14)=1.45, p>$ $.05]$ of the three-dimensional pyramid. Similarly, no differences were present across conditions for the pretest measurements of the binocular $[F(2,14)=1.64, p>.05]$ or monocular $[F(2,14)=0.48, p>.05]$ apparent distance of the two-dimensional target or pretest measurements of lateral phoria $[F(2,14)=1.74, p>.05]$.

Analysis of the aftereffect scores indicated that a significant reduction in apparent depth occurred under both the kinetic (standard) $[t(7)=-5.93, p \leq .05]$ and static exposure conditions $[t(7)=-5.27, p \leq .05]$. In the artificial-pupil condition, a small but nonsignificant increase in apparent depth was found $[t(7)=1.88, p>$ $.05]$. As might be expected, the magnitude of the depth aftereffect differed significantly across conditions $[F(2,14)=27.64, p \leq .01]$, being greater in the kinetic and static conditions than in the pupil condition $[t(7)=$ $-7.13, p \leq .05$, and $t(7)=-4.46, p \leq .05$, respectively]. No difference was found between the kinetic and static conditions $[t(7)=-2.12, p>.05]$. These results indicate that kinetic depth information is not necessary for the adaptation effect to occur. Moreover, the absence of any change under the artificial-pupil condition suggests that the depth aftereffect depends on active maintenance of accommodation during the exposure interval.

Differences in the magnitude of the binocular distance aftereffect were found across conditions when the threedimensional pyramid was used as the target $[F(2,14)=$ $4.28, p \leq .05]$. The changes produced under the kinetic and static conditions exceeded that produced under the pupil condition $[t(7)=-2.57, p \leq .05$ and $t(7)=$ $-2.56, p \leq .05$, respectively]. The reduction in binocular apparent distance was significant under the kinetic $[t(7)=-5.51, p \leq .05]$ and static conditions $[t(7)=$ $-5.76, p \leq .05]$, but not under the artificial-pupil condition $[t(7)=-1.45, p>.05]$. The pattern of results was therefore similar to that found for the depth aftereffect.

The distance aftereffects measured using the twodimensional target differed depending on whether testing was carried out binocularly or monocularly. Under binocular conditions, no differences were found across conditions $[F(2,14)=0.54, p>.05]$; the distance aftereffects were small and nonsignificant in each case. With monocular testing, however, the magnitude of the distance aftereffect differed across conditions $[F(2,14)=12.11$, $p \leq .05]$. The change in monocular apparent distance was greater in the kinetic condition than in the artificial-pupil condition $[t(7)=-2.89, p \leq .05]$ and greater in the static than in the artificial-pupil condition $[t(7)=-4.26, p \leq$ $.05]$. Both kinetic and static exposure produced significant reductions in monocular apparent distance. In contrast, the small increase in monocular apparent distance measured under the artificial-pupil condition failed to differ significantly from zero $[t(7)=0.66, p>.05]$. The monocular distance results may indirectly implicate processes within the accommodative system.

There were no differences across conditions with respect to changes in apparent size $[F(2,14)=0.41, p>$ $.05]$ or phoria $[F(2,14)=0.19, p>.05]$. Both the size

Table 2

Means and Standard Errors for Measurements in Experiment 2

\begin{tabular}{|c|c|c|c|c|c|c|c|c|c|c|}
\hline \multirow[b]{2}{*}{ Test Type } & & \multicolumn{3}{|c|}{ Kinetic Exposure } & \multicolumn{3}{|c|}{ Static Exposure } & \multicolumn{3}{|c|}{ Pupil Exposure } \\
\hline & & Pretest & Posttest & $\Delta$ & Pretest & Posttest & $\Delta$ & Pretest & Posttest & $\Delta$ \\
\hline Depth & $\begin{array}{l}\text { Mean } \\
S E\end{array}$ & $\begin{array}{l}8.07 \\
0.60\end{array}$ & $\begin{array}{l}6.29 \\
0.80\end{array}$ & $\begin{array}{c}-1.78^{*} \\
0.30\end{array}$ & $\begin{array}{l}8.50 \\
0.82\end{array}$ & $\begin{array}{l}7.71 \\
0.80\end{array}$ & $\begin{array}{l}-0.79 * \\
0.15\end{array}$ & $\begin{array}{l}8.76 \\
0.78\end{array}$ & $\begin{array}{l}9.21 \\
0.89\end{array}$ & $\begin{array}{l}0.45 \\
0.24\end{array}$ \\
\hline $\begin{array}{l}\text { Binocular Distance } \\
\text { (Pyramid) }\end{array}$ & $\begin{array}{l}\text { Mean } \\
S E\end{array}$ & $\begin{array}{r}41.82 \\
1.37\end{array}$ & $\begin{array}{r}39.01 \\
1.11\end{array}$ & $\begin{array}{c}-2.81^{*} \\
0.51\end{array}$ & $\begin{array}{r}44.00 \\
1.56\end{array}$ & $\begin{array}{r}40.89 \\
1.48\end{array}$ & $\begin{array}{c}-3.11^{*} \\
0.54\end{array}$ & $\begin{array}{r}44.25 \\
1.74\end{array}$ & $\begin{array}{r}43.32 \\
2.16\end{array}$ & $\begin{array}{r}-0.93 \\
0.64\end{array}$ \\
\hline $\begin{array}{l}\text { Binocular Distance } \\
\text { (Two-dimensional Target) }\end{array}$ & $\begin{array}{l}\text { Mean } \\
S E\end{array}$ & $\begin{array}{r}37.64 \\
1.42\end{array}$ & $\begin{array}{r}36.97 \\
2.08\end{array}$ & $\begin{array}{r}-0.67 \\
1.42\end{array}$ & $\begin{array}{r}39.99 \\
1.94\end{array}$ & $\begin{array}{r}39.38 \\
2.12\end{array}$ & $\begin{array}{r}-0.61 \\
0.99\end{array}$ & $\begin{array}{r}40.15 \\
2.30\end{array}$ & $\begin{array}{r}40.91 \\
2.18\end{array}$ & $\begin{array}{l}0.76 \\
0.87\end{array}$ \\
\hline $\begin{array}{l}\text { Monocular Distance } \\
\text { (Two-dimensional Target) }\end{array}$ & $\begin{array}{l}\text { Mean } \\
S E\end{array}$ & $\begin{array}{r}40.68 \\
1.80\end{array}$ & $\begin{array}{r}37.32 \\
1.69\end{array}$ & $\begin{array}{l}-3.36^{*} \\
0.50\end{array}$ & $\begin{array}{r}41.96 \\
2.12\end{array}$ & $\begin{array}{r}39.61 \\
2.15\end{array}$ & $\begin{array}{l}-2.35 * \\
0.57\end{array}$ & $\begin{array}{r}42.24 \\
2.49\end{array}$ & $\begin{array}{r}42.97 \\
3.01\end{array}$ & $\begin{array}{l}0.73 \\
1.11\end{array}$ \\
\hline Size & $\begin{array}{l}\text { Mean } \\
S E\end{array}$ & $\begin{array}{l}5.00 \\
0.24\end{array}$ & $\begin{array}{l}5.29 \\
0.46\end{array}$ & $\begin{array}{l}0.29 \\
0.27\end{array}$ & $\begin{array}{l}5.46 \\
0.47\end{array}$ & $\begin{array}{l}5.50 \\
0.54\end{array}$ & $\begin{array}{l}0.04 \\
0.19\end{array}$ & $\begin{array}{l}5.57 \\
0.52\end{array}$ & $\begin{array}{l}5.66 \\
0.64\end{array}$ & $\begin{array}{l}0.09 \\
0.22\end{array}$ \\
\hline Phoria & $\begin{array}{l}\text { Mean } \\
S E\end{array}$ & $\begin{array}{r}-10.38 \\
1.75\end{array}$ & $\begin{array}{r}-10.25 \\
1.98\end{array}$ & $\begin{array}{l}0.13 \\
0.79\end{array}$ & $\begin{array}{r}-9.50 \\
1.88\end{array}$ & $\begin{array}{r}-9.50 \\
2.59\end{array}$ & $\begin{array}{l}0.00 \\
1.19\end{array}$ & $\begin{array}{r}-9.19 \\
1.89\end{array}$ & $\begin{array}{r}-9.63 \\
2.23\end{array}$ & $\begin{array}{r}-0.44 \\
0.93\end{array}$ \\
\hline
\end{tabular}

*Significant at the $p=.05$ level or better. Apparent depth, distance and size scores are in centimeters. Phoria scores are in prism diopters; negative phoria scores indicate exophoria. Aftereffect values $(\Delta)$ are derived from the (posttest - pretest) scores for each subject. 
aftereffect and the phoria shift measured under each condition proved minimal and nonsignificant.

\section{EXPERIMENT 3}

One possible problem with the use of artificial pupils during exposure to the telestereoscope is that the small apertures may alter the stimulus situation in some way, rather than simply reducing the need for active accommodation. Biersdorf and Baird (1966) reported a $10 \%$ reduction in image size with an artificial pupil of the same size $(2 \mathrm{~mm})$ placed at about the same distance from the eye $(2 \mathrm{~cm})$ as in Experiment 2 of the present study. A reduction in image size would have the same effect on binocular disparity as would an increase in viewing distance for two points separated by a constant depth interval: the magnitude of the disparity between the images in the two eyes would be somewhat smaller than with standard exposure. Since the degree of conflict between disparity and other sources of depth information would be diminished, at least some of the basis for a reduction in apparent depth via recalibration would be removed. It might be argued, therefore, that the difference in results under the standard and artificial-pupil conditions is a reflection of the difference in disparity in the two cases, rather than the effect of lessening the accommodative innervation necessary for sharp focus.

In Experiment 3, the effects of binocular observation of the rotating form through the mirrors of the telestereoscope with and without artificial pupils were again compared. Control of image size was achieved by using an exposure form for the artificial-pupil condition that was $10 \%$ larger than that used for the standard condition. As a precaution, the apparent size of each form was also checked under the corresponding conditions.

\footnotetext{
Method

Design and Procedure. The two exposure conditions, standard and artificial-pupil, were tested in a within-subjects design. The dependent measure consisted of the change in apparent depth (posttest - pretest). Separate sessions, separated by a minimum of $24 \mathrm{~h}$, were used for each exposure condition. The order of the standard and artificial-pupil conditions was counterbalanced across subjects so that half received the standard condition first and half received the artificial-pupil condition first.

Lateral phoria was first measured with the Maddox rod. The subjects then made four distance judgments using the two-dimensional target. These corresponded to the practice and pretest distance measures taken in Experiments 1 and 2. The distance tests were included in order to keep all the criteria for screening subjects the same as those used in the preceding experiments. Performance was considered acceptable if apparent distance consistently increased with target distance.

Following the distance and phoria measurements, depth pretests were carried out with the pyramid target in the same manner as in the earlier experiments. The subject was then fitted with trial frames containing either 0.0 SD lenses or artificial pupils. The threedimensional pyramid was removed and replaced by the cube (large for the artificial-pupil condition and small for the $0.0 \mathrm{SD}$ lens condition) to be used as the exposure target for that particular session. The cube was stationary initially and was placed so that its nearest
}

face would be parallel to the subject's frontal plane. The subject made two judgments of the size of the nearest face along the diagonal. The size tests were included as a check on the equivalence of the apparent sizes of the large and small cubes as seen through the pupils and $0.0 \mathrm{SD}$ lenses, respectively. The cube was then set into rotation and observed for a 10 -minute interval, after which the depth tests (pyramid target) were repeated.

Subjects. Eight female and two male undergraduate students ranging in age from 18 to 21 served as subjects. Eight additional subjects were dismissed from the experiment for various reasons. One subject had poor acuity (20/40) in one eye. Two subjects could not maintain fusion of the pyramid during the pretests. The remaining five subjects were dismissed because they either showed reverse adaptation or unacceptably small reductions in apparent depth as a result of standard binocular exposure to the telestereoscope.

\section{Results}

No difference was found between the pretest depth judgments under the standard $($ mean $=11.27 \mathrm{~cm}$ ) and artificial-pupil (mean $=11.41 \mathrm{~cm})$ conditions $[t(9)=$ $-0.21, p>.05]$. The apparent size of the exposure form averaged $7.85 \mathrm{~cm}$ with the 0.0 SD lenses and $8.03 \mathrm{~cm}$ with the artificial pupils; the difference of $0.18 \mathrm{~cm}$ was nonsignificant $[t(9)=0.85, p>.05]$. It can therefore be inferred that the larger size of the exposure form used in the artificial-pupil condition was effective in counteracting any effect of the small aperture on image size.

Following exposure, apparent depth averaged $9.69 \mathrm{~cm}$ for the standard condition and $11.91 \mathrm{~cm}$ for the pupil condition. This amounted to a significant reduction in apparent depth of $-1.58 \mathrm{~cm}$ in the former condition $[t(9)=$ $-5.85, p \leq .05]$ and a small but nonsignificant increase of $+0.73 \mathrm{~cm}$ in the latter condition $[t(9)=1.79, p>$ $.05]$.

\section{GENERAL DISCUSSION}

The results of the present series of experiments provide strong evidence against the recalibration of disparity by kinetic depth as the mechanism underlying adaptation to the telestereoscope. The conflict between these cues is not necessary to produce the effect, since a significant reduction in apparent depth can be obtained under conditions in which either disparity (Experiment 1) or kinetic depth (Experiment 2) is absent. The existence of the depth aftereffect following monocular exposure is especially noteworthy because binocular disparity was not even present as a depth cue during the viewing period. The change in depth perception in this case cannot be attributed to the recalibration of disparity by veridical, nonkinetic sources of depth information (i.e., cues such as linear perspective), since monocular exposure precludes any form of cue conflict.

No change in depth perception was found when artificial pupils were worn while observing the rotating object through the mirrors (Experiment 2). Moreover, the same result was obtained when no difference in image size existed between the standard and artificial-pupil conditions (Experiment 3). When image size is controlled, the degree of cue conflict inherent in the telestereoscope situation is 
the same, regardless of whether or not the form is seen through a small aperture.

The experiments with the artificial pupils demonstrated that cue conflict is not itself sufficient to produce the adaptation effect. Given that the critical feature on which recalibration is based is neither necessary nor sufficient to produce the adaptation effect, there is little reason to utilize recalibration as an explanatory mechanism. Moreover, there were additional results that point toward processes other than recalibration.

Subjects made judgments of both the apparent depth and the apparent distance of the three-dimensional test target in Experiments 1 and 2. Whenever a large depth aftereffect was produced, it was always associated with a significant decrease in the apparent distance of the form. Since the target must necessarily appear reduced in depth if it is seen as nearer than before, any additional process involving recalibration of binocular disparity becomes superfluous for the most part. Although perceived distance may not precisely correspond to the distance registered with regard to depth processing, the former may be used as an estimate of the latter. With disparity held constant, perceived depth varies as a function of distance squared. Hence, the proportional relationship between pre- and posttest depth scores should be approximately equivalent to the square of that between pre- and posttest distance scores. Applying this procedure, the reduction in perceived distance appears to be capable of accounting for about $88 \%$ and $64 \%$ of the depth aftereffect obtained in the standard exposure condition in Experiments 1 and 2, respectively. Clearly, by this account alone, the role of recalibration (if any) in the production of the adaptation effect need not be very great.

One result that appears to be inconsistent with the hypothesis that exposure to the telestereoscope primarily affects the registration of distance is the failure to obtain a change in the apparent distance of the two-dimensional target comparable in magnitude to that found for the threedimensional pyramid under similar test conditions (binocular viewing). However, this result may be understandable if the perceptual aftereffects are ultimately found to derive from oculomotor adaptation. Given that the apparent distance of the two-dimensional target was assessed near the end of the posttest sequence, any underlying oculomotor changes might be attenuated as a result of decay and/or the influence of the interpolated measurements using the pyramid target. ${ }^{5}$

Previous investigators (e.g., Wallach \& Karsh, 1963) concluded from the absence of a size aftereffect that the reduction in apparent depth could not possibly be due to a change in registered distance. The present experiments show, however, that apparent size is inadequate as an index of the change in distance perception, since no size aftereffect was ever obtained despite significant reductions in apparent distance.

Several forms of converging evidence point toward an accommodative locus for the cause of the perceptual aftereffects. Different results are obtained depending on whether fusional vergence input is absent or accommodative demand is reduced during the inducing interval. With the fusional system in the open-loop mode (monocular exposure), a significant depth aftereffect is produced. However, when the accommodative demand is reduced during exposure (binocular viewing with artificial pupils), the depth aftereffect is entirely absent.

The occurrence of changes in perceived distance and the absence of changes in phoria support the idea that the perceptual aftereffects do not result from changes in tonic vergence. The reduction in apparent depth following standard exposure is accompanied by a change in monocular apparent distance (Experiments 1 and 2) that is significantly larger than the corresponding binocular distance aftereffect for the same target. No measurable shift in lateral phoria was found to occur in conjunction with the change in binocular apparent distance. This contrasts sharply with the pattern normally associated with fusional hysteresis, that is, an induced phoria and a change in apparent distance that is larger binocularly than monocularly (Ebenholtz \& Fisher, 1982).

Further research is required to determine whether the perceptual aftereffects could derive from other forms of oculomotor change linked to the accommodative system. For example, Miles and Judge (1982) reported that the relationship between accommodation and accommodative vergence is altered following exposure to an optical device similar to the telestereoscope. An increase was found in the degree of accommodative vergence produced in conjunction with a given level of accommodative response, that is, in the response AC/A ratio. Additional experiments are necessary to determine whether the perceptual aftereffects of distance and depth result from such adaptation of the cross-linkage gain between the accommodation and vergence systems.

\section{REFERENCES}

BIERSDORF, W. R., BAIRD, J. C. (1966). Effects of an artificial pupil and accommodation on retinal image size. Journal of the Optical Society of America, 56, 1123-1129.

DUKE-ELDER, S., \& WYBAR, K. (1973). Ocular motility and strabismus. In S. Duke-Elder (Ed.), System of ophthalmology (Vol. 6). St. Louis: Mosby.

EBENHOLTz, S. M. (1983). Accommodative hysteresis: A precursor for induced myopia? Investigative Ophthalmology \& Visual Science, 24, 513-515.

EBENHOLTZ, S. M. (1985). Accommodative hysteresis: Relation to resting focus. American Journal of Optometry \& Physiological Optics, 62, 755-762.

EbEnholtz, S. M., Fisher, S. K. (1982). Distance adaptation depends upon plasticity in the oculomotor control system. Perception \& Psychophysics, 31, 551-560.

Miles, F. A., JUDGE, S. J. (1982). Optically-induced changes in the neural coupling between vergence eye movements and accommodation in human subjects. In G. Lennerstrand, D. S. Zee, \& E. Keller (Eds.), Functional aspects of ocular motility disorders. Oxford: Pergamon.

ONo, H., \& COMERFord, J. (1977). Stereoscopic depth constancy. In W. Epstein (Ed.), Stability and constancy in visual perception: Mechanisms and processes. New York: Wiley.

Ripps, H., Chin, N. B., Siegel, I. M., \& Breinin, G. M. (1962). The 
effect of pupil size on accommodation, convergence and the AC/A ratio. Investigative Ophthalmology \& Visual Science, 1, 127-135.

RITTER, M. (1979). Perception of depth: Processing of simple and positional disparities as a function of viewing distance. Perception \& Psychophysics, 25, 209-214.

Wallach, H. (1976). On perception. New York: Quadrangle.

Wallach, H., FreY, K., BoDE, K. (1972). The nature of adaptation in distance perception based on oculomotor cues. Perception \& Psychophysics, 11, 110-116.

WALLACH, H., \& KARSH, E. (1963). The modification of stereoscopic depth perception and the kinetic depth-effect. American Journal of Psychology, 76, 429-435.

WALLACH, H., MOORE, M., \& DAvidson, L. (1963). Modification of stereoscopic depth perception. American Journal of Psychology, 76, 191-204.

WALLACH, H., \& O'ConNELL, D. N. (1953). The kinetic depth effect. Journal of Experimental Psychology, 45, 205-217.

WALLACH, H., ZUCKERMAN, C. (1963). The constancy of stereoscopic depth. The American Journal of Psychology, 76, 404-412.

WARD, P. A., \& Charman, W. N. (1985). Effect of pupil size on steady state accommodation. Vision Research, 25, 1317-1326.

\section{NOTES}

1. Nonkinetic sources of veridical depth information (e.g., linear perspective) are also present when a three-dimensional form is viewed through the telestereoscope. Such cues could potentially be effective in recalibrating binocular disparity. The adaptive pre- to posttest reduc- tion in apparent depth that has been found following observation of a static, rather than rotating, form during the exposure interval has previously been accounted for in these terms (Wallach \& Karsh, 1963; Wallach, Moore \& Davidson, 1963).

2. The magnitude and direction of the conflict between the stimuli for accommodation and convergence depends on the following parameters: the angle and separation of one set of mirrors relative to the other, the physical depth and distance of the form seen through the mirrors, and the observer's interocular separation and locus of fixation.

3. Although the demand for accommodation may not have been eliminated completely, there is little doubt that the $2-\mathrm{mm}$ pupil significantly reduced blur-driven accommodation (Ripps, Chin, Siegel, \& Breinin, 1962; Ward \& Charman, 1985).

4. Although a chin and forehead rest was used, some slight head movements may nevertheless have occurred during the exposure period. The resultant motion parallax would constitute a veridical depth cue and would therefore be expected to enhance recalibration.

5. In a preliminary experiment in which distance judgments were made only for the two-dimensional target, a significant reduction in binocular apparent distance, comparable in magnitude to that reported here for the three-dimensional pyramid, was consistently found. These results lend support to the idea that the distance aftereffects for the twodimensional target in the present study were greatly reduced either because of decay over time or because of the intervening posttests with the pyramid target.

(Manuscript received July 26, 1985; revision accepted for publication June 13, 1986.) 